\title{
ON A RECENTLY PROPOSED RELATION BETWEEN oHS AND ITO SYSTEMS
}

\author{
Atalay Karasu \\ Department of Physics, Faculty of Arts and Sciences \\ Middle East Technical University , 06531 Ankara-Turkey
}

\begin{abstract}
The bi-Hamiltonian structure of original Hirota-Satsuma system proposed by Roy based on a modification of the bi-Hamiltonian structure of Ito system is incorrect.
\end{abstract}


In a recent paper [1] a bi-Hamiltonian structure for the original HirotaSatsuma(oHS) system where $a$ is arbitrary is proposed and a relation between oHS and Ito systems is introduced and a recursion operator is found for the oHS system. In this note we point out that the oHS system does not in fact admit a bi-Hamiltonian structure and the relation between the oHS and Ito systems claimed by the author of [1] is actually incorrect.

It is well known that the original Hirota-Satsuma system (oHS) [2]

$$
\begin{aligned}
& u_{t}=a\left(u_{x x x}+6 u u_{x}\right)+2 b v v_{x} \\
& v_{t}=-v_{x x x}-3 u v_{x}
\end{aligned}
$$

for all values of $a$ and $b$, possesses three conserved quantities.

$$
I_{1}=u, \quad I_{2}=u^{2}+\frac{2}{3} b v^{2}, \quad I_{3}=(1+a)\left(u^{3}-\frac{1}{2}\left(u_{x}\right)^{2}\right)+b\left(u v^{2}-\left(v_{x}\right)^{2}\right) .
$$

Later Hirota-Satsuma [3] showed that oHS system has infinitely many conserved quantities for the choice of $a=\frac{1}{2}$ and conjectured that it is completely integrable. Dodd and Fordy 四 showed that the oHS system admits a Lax representation only for this particular value of $a=\frac{1}{2}$. Also Aiyer [5] proved that the oHS system possesses a recursion operator of degree four only for $a=\frac{1}{2}$. The same result has been recently reported by the author and Gürses [6] - [8] in the context of the integrable coupled KdV systems admitting recursion operators. Wilson [9] pointed out that the oHS system with $a=\frac{1}{2}$ belongs to the general construction of evolutionary equations possessesing Lax-pair due to Drinfeld and Sokolov [10]-[11]. On the other hand the Ito system 12

$$
u_{t}=u_{x x x}+6 u u_{x}+2 v v_{x},
$$




$$
v_{t}=2(u v)_{x} .
$$

admits a bi-Hamiltonian structure

$$
B_{I I} \delta \mathcal{H}_{n}=B_{I} \delta \mathcal{H}_{n+1}
$$

where

$$
B_{I I}=\left(\begin{array}{cc}
D^{3}+4 u D+2 u_{x} & 2 v D \\
2 v_{x}+2 v D & 0
\end{array}\right), B_{I}=\left(\begin{array}{cc}
D & 0 \\
0 & D
\end{array}\right) .
$$

with the Hamiltonian functionals

$$
\begin{aligned}
\mathcal{H}_{1}[u, v] & =\int \frac{1}{2}\left(u^{2}+v^{2}\right) d x, \\
\mathcal{H}_{2}[u, v] & =\int \frac{1}{2}\left(u^{3}-\frac{1}{2} u_{x}^{2}+u v^{2}\right) d x .
\end{aligned}
$$

The recursion operator arising from a Hamiltonian pair

$$
R=B_{I I}\left(B_{I}\right)^{-1}=\left(\begin{array}{cc}
D^{2}+4 u+2 u_{x} D^{-1} & 2 v \\
2 v+2 v_{x} D^{-1} & 0
\end{array}\right) .
$$

is a hereditary operator [7] which gives rise to infinitely many conserved quantities. The multi-Hamiltonian structure of this system was studied by Antonowicz and Fordy [13] and by Olver and Rosenau [14].

At this stage we have the following observations:

observation 1:

The author of [1] points out that there is a printing error in the conserved density $I_{3}$ (instead of $1+a$ there is $a$ ) obtained in [2] . But this claim is wrong and $I_{3}$ in the ref. [3] is correct as it is. One can check this either using $\frac{d}{d t} \int I_{3} d x=0$ or finding its gradient $\gamma_{3}$ satisfies $\gamma_{3}^{\prime}[K]+\left(K^{\prime}\right)^{\dagger}\left[\gamma_{3}\right]=0$ and $\gamma_{3}^{\prime}=\left(\gamma_{3}^{\prime}\right)^{\dagger}[15$. Here $K$ is the right hand side of the oHS system. 
observation 2:

The author of [1] claims that the oHS system can be written as

$$
\left(\begin{array}{l}
u \\
v
\end{array}\right)_{t}=A B_{1}\left(\begin{array}{c}
\frac{\delta \mathcal{H}_{1}}{\delta u} \\
\frac{\delta \mathcal{H}_{1}}{\delta v}
\end{array}\right)
$$

with

$$
A=\left(\begin{array}{cc}
a\left(D^{2}+4 u+2 u_{x} D^{-1}\right) & 2 v+v_{x} D^{-1} \\
2 v+v_{x} D^{-1} & -\left(D^{2}+4 u+2 u_{x} D^{-1}\right)
\end{array}\right)
$$

and $B_{I}$ given in (5). Here $\mathcal{H}_{1}$ is the Hamiltonian functional of the Ito system. First we have noticed that $\mathcal{H}_{1}$ is also a Hamiltonian functional of the oHS system $(b=3 / 2)$. Second, the operator $A B_{1}$ does not satisfy the Jacobi identity [16]. Therefore it is not a Hamiltonian operator for the oHS system although it is a skew-symmetric operator. Furthermore the author of [1] proposes a bi-Hamiltonian structure for the oHS system which is based on the bi-Hamiltonian form of Ito system (4) as

$$
A B_{I I} \delta \mathcal{H}_{n}=A B_{I} \delta \mathcal{H}_{n+1}, \quad n=0,1,2, \ldots
$$

First of all,the Hamiltonian functional $\mathcal{H}_{2}$ of the Ito system is not a Hamiltonian functional of the oHS system. Moreover neither $A B_{I}$ nor $A B_{I I}$ are Hamiltonian operators. As a result the expression (10) does not constitute a bi-Hamiltonian form for the oHS system.

observation 3

The author of [1] claims that an infinite hierarchy of the oHS system is generated by the recursion operator

$$
\mathcal{R}=\left(A B_{I I}\right)\left(A B_{I}\right)^{-1}=A R A^{-1}
$$


where $\mathcal{R}$ is the recursion operator for Ito system. It is easy to see that the given recursion operator $\mathcal{R}$ has at most degree two. Therefore it does not generate the hierarchy of the oHS system. It has been recently reported that neither oHS nor HS with $a=\frac{1}{2}$ possess a recursion operator of degree two [6]-[8].

As a conclusion, contrary to the claims made in ref.[四], the oHS system is not a bi-Hamiltonian system. This system is integrable and admits a biHamiltonian structure only when $a=\frac{1}{2}[17]-[18]$. The hierarchy of this system was studied by Levi [19].

The author would like to thank M. Gürses for bringing this recent work on Hirota-Satsuma system to my attention and for many valuable discussions. This work is partially supported by the Scientific and Technical Research Council of Turkey (TUBITAK).

\section{References}

[1] P.K. Roy , Phys.Lett. A,249, (1998) 55.

[2] R. Hirota and J. Satsuma, Phys.Lett. A,85, (1981) 407.

[3] J. Satsuma and R. Hirota ,J. Phys.Soc.Japan 51, (1982) 3390.

[4] R. Dodd and A.P. Fordy, Phys.Lett. A,89, (1982) 168.

[5] R.N. Aiyer, Phys.Lett.A,93,(1983) 368.

[6] M. Gürses and A. Karasu, Phys.Lett. A,214, (1996) 21.

[7] M. Gürses and A. Karasu, J.Math.Phys.,39, (1998) 2103. 
[8] M. Gürses and A. Karasu Phys.Lett. A,251, (1999) 247.

[9] G. Wilson, Phys.Lett. A,89, (1982) 332.

[10] V.G.Drinfeld and V.V Sokolov, Proc. S.L. Sobolev Seminar,Novosibirsk,Vol.2 (1981) pp.5-9 (in Russian).

[11] V.G.Drinfeld and V.V Sokolov, J.Sov.Math.30, (1985) 1975.

[12] M. Ito , Phys.Lett.A,91,(1982) 353.

[13] M.Antonowicz and A.P.Fordy, Physica D 28, (1987) 345.

[14] P.J.Olver and P.Rosenau, Phys.Rev.E 53, (1996) 1900.

[15] B. Fuchssteiner and A.S.Fokas, Physica D,4, (1981) 47.

[16] P.J. Olver. Applications of Lie Groups to Differential Equations, 2nd Ed., Graduate Texts in Mathematics, Vol.107 (Springer, New York, 1993).

[17] B. Fuchssteiner, Prog.Theor.Phys.,68, (1982) 1082 .

[18] W. Oevel, Phys.Lett.A,94, (1983) 404.

[19] D. Levi, Phys.Lett.A,95, (1983) 7. 